\title{
Spectral Stability of Stationary Solutions of a Boussinesq System Describing Long Waves in Dispersive Media*
}

\author{
Min Chen ${ }^{\dagger}$, Christopher W. Curtis $\ddagger^{\ddagger}$, Bernard Deconinck ${ }^{\S}$, Crystal W. Lee ${ }^{\ddagger}$, and \\ Nghiem Nguyen
}

\begin{abstract}
We study the spectral (in)stability of one-dimensional solitary and cnoidal waves of various Boussinesq systems. These systems model three-dimensional water waves (i.e., the surface is two-dimensional) with or without surface tension. We present the results of numerous computations examining the spectra related to the linear stability problem for both stationary solitary and cnoidal waves with various amplitudes, as well as multipulse solutions. The one-dimensional nature of the wave forms allows us to separate the dependence of the perturbations on the spatial variables by transverse wave number. The compilation of these results gives a full view of the two-dimensional stability problem of these one-dimensional solutions. We demonstrate that line solitary waves with elevated profiles are spectrally stable with respect to one-dimensional perturbations and long transverse perturbations. We show that depression solitary waves are spectrally stable with respect to one-dimensional perturbations, but unstable with respect to transverse perturbations. We also discuss the instability of multipulse solitary waves and cnoidal-wave solutions of the Boussinesq system.
\end{abstract}

Key words. water waves, Boussinesq system, spectral stability, transverse perturbation, solitary waves, cnoidal waves

AMS subject classifications. 76B15, 35P99, 34D20, 65P30

DOI. $10.1137 / 090779929$

1. Introduction. In [3, 4] Bona, Chen, and Saut derive a three-parameter family of Boussinesq equations to describe the dynamics of small-amplitude long waves on the surface of an ideal fluid as governed by the inviscid Euler equations. In [3] the different model equations are derived and their well-posedness around the rest state is examined in a variety of function spaces. In [4] various results extend the linear analysis of [3] to the nonlinear regime. The three-dimensional versions of the equations derived by Bona, Chen, and Saut are presented explicitly and analyzed in [5]. These systems of equations describe a two-dimensional water

\footnotetext{
${ }^{*}$ Received by the editors December 11, 2009; accepted for publication (in revised form) by C. Wayne June 23, 2010; published electronically September 7, 2010. Any opinions, findings, and conclusions or recommendations expressed in this material are those of the authors and do not necessarily reflect the views of the funding sources.

http://www.siam.org/journals/siads/9-3/77992.html

${ }^{\dagger}$ Department of Mathematics, Purdue University, West Lafayette, IN 47907 (chen45@purdue.edu).

${ }^{\ddagger}$ Department of Applied Mathematics, University of Colorado, Boulder, CO 80309-0526 (christopher.w.curtis@ colorado.edu, crystal.w.lee@hotmail.com). The second author was partly supported by the National Science Foundation under grant NSF-DMS-VIGRE-0354131.

${ }^{\S}$ Department of Applied Mathematics, University of Washington, Seattle, WA 98195-2420 (bernard@math. washington.edu). This author was partly supported by the National Science Foundation under grant NSF-DMS0604546.

"Department of Mathematics and Statistics, Utah State University, Logan, UT 84322-3900 (nghiem.nguyen@ usu.edu). This author was partly supported by the National Science Foundation under grant NSF-DMS-0402815 (as an NSF Postdoctoral Fellow).
} 
wave surface; see (1.1) below. In [8] Chen, and in a subsequent paper [10] Chen, Chen, and Nguyen, were able to provide an explicit description for different branches of exact solutions of the various Boussinesq equations. All the solutions that they constructed are one-dimensional solutions, in the sense that in a suitably rotated frame the solutions depend only on the first spatial variable, $x$, and not on the second one, $y$. We refer to such solutions as line solutions. Chen, Chen, and Nguyen were able to construct both periodic and solitary wave solutions. The periodic solutions are referred to as cnoidal wave solutions, since their explicit description may be given in terms of the Jacobi elliptic functions. However, a more transparent description may be given as well, using Weierstrass elliptic functions. That description is given in Appendix B.

In this paper we return to the result of [10] in order to examine the spectral stability of the different line solutions constructed there. We investigate the stability both with respect to onedimensional perturbations (depending only on $x$ ) and with respect to fully two-dimensional perturbations (characterized by a wave length in the $y$ direction). Due to the complicated nature of the equations, we restrict ourselves to numerical investigations for now, using Hill's method [14] or an extension of it to the whole line using Hermite functions. This method is described in Appendix A.

The three-parameter family of Boussinesq equations whose solutions we will examine is given by

$$
\begin{aligned}
& \eta_{t}+\nabla \cdot \mathbf{v}+\nabla \cdot(\eta \mathbf{v})+a \nabla \cdot \Delta \mathbf{v}-b \Delta \eta_{t}=0 \\
& \mathbf{v}_{t}+\nabla \eta+\frac{1}{2} \nabla \mathbf{v}^{2}+c \nabla(\Delta \eta)-d \Delta \mathbf{v}_{t}=0 .
\end{aligned}
$$

Here $\nabla=\left(\partial_{x}, \partial_{y}\right)^{T}$, with $x$ and $y$ the spatial independent variables. The temporal independent variable is denoted by $t$. The dependent variables are denoted by $\eta$, the scaled wave height, and $\mathbf{v}$, a scaling of the fluid velocity. Further, $a, b, c$, and $d$ are parameters, related by [3]

$$
a+b=\frac{1}{2}\left(\theta^{2}-\frac{1}{3}\right), \quad c+d=\frac{1}{2}\left(1-\theta^{2}\right),
$$

where $\theta \in[0,1]$ is a fixed constant. Since $a+b+c+d=1 / 3$, none of the situations that we consider in this paper take surface tension into account [3].

In order to study the linear stability of traveling line solitary waves and line cnoidal wave solutions under the influence of two-dimensional small perturbations, we move to a frame translating with velocity $W$ in the $x$ direction,

$$
\hat{x}=x-W t, \quad \hat{y}=y, \quad \hat{t}=t,
$$

so that

$$
\partial_{x}=\partial_{\hat{x}}, \quad \partial_{y}=\partial_{\hat{y}}, \quad \partial_{t}=\partial_{\hat{t}}-W \partial_{\hat{x}} .
$$

In what follows, we omit the ^ to simplify the notation. In this translating frame, equations (1.1) become

$$
\begin{aligned}
& \eta_{t}-W \eta_{x}+\nabla \cdot \mathbf{v}+\nabla \cdot(\eta \mathbf{v})+a \nabla \cdot \Delta \mathbf{v}-b \Delta \eta_{t}+b W \Delta \eta_{x}=0 \\
& \mathbf{v}_{t}-W \mathbf{v}_{x}+\nabla \eta+\frac{1}{2} \nabla \mathbf{v}^{2}+c \nabla(\Delta \eta)-d \Delta \mathbf{v}_{t}+d W \Delta \mathbf{v}_{x}=0 .
\end{aligned}
$$


In lieu of nonlinear or orbital stability, we limit our considerations to those of spectral stability. To this end, we linearize the above equations around the stationary line solution $\left(\eta^{*}, u^{*}, 0\right)$ (where $\eta^{*}$ and $u^{*}$ are independent of $y$ ) of (1.5), i.e.,

$$
\eta^{*}(x, y, t)=\eta(x), \quad u^{*}(x, y, t)=u(x),
$$

where one should recall that we earlier transformed to a translating frame of reference. In order to linearize, we assume solutions of the form

$$
\begin{aligned}
& \eta(x, y, t)=\eta^{*}+\epsilon \bar{\eta}(x, y, t)+\mathcal{O}\left(\epsilon^{2}\right), \\
& u(x, y, t)=u^{*}+\epsilon \bar{u}(x, y, t)+\mathcal{O}\left(\epsilon^{2}\right), \\
& v(x, y, t)=0+\epsilon \bar{v}(x, y, t)+\mathcal{O}\left(\epsilon^{2}\right) .
\end{aligned}
$$

By substituting (1.6) into (1.5) and dropping terms of order $\epsilon^{2}$, a system of linear equations governing the dynamics of $(\bar{\eta}, \bar{u}, \bar{v})$ is obtained. Dropping the bars for simplicity of notation, we have

$$
\begin{aligned}
& -\eta_{t}+b \Delta \eta_{t}=-W \eta_{x}+b W \Delta \eta_{x}+\nabla \cdot \mathbf{v}+a \nabla \cdot \Delta \mathbf{v}+\nabla \cdot\left(\eta^{*} \mathbf{v}+\mathbf{v}^{*} \eta\right) \\
& -\mathbf{v}_{t}+d \Delta \mathbf{v}_{t}=-W \mathbf{v}_{x}+d W \Delta \mathbf{v}_{x}+\nabla \eta+c \nabla(\Delta \eta)+\nabla\left(\mathbf{v} \cdot \mathbf{v}^{*}\right)
\end{aligned}
$$

where $\mathbf{v}^{*}=\left(u^{*}, 0\right)^{T}$.

Since these linear equations are autonomous in both $y$ and $t$, we separate the dependence on the independent variables as follows:

$$
\begin{aligned}
& \eta(x, y, t)=N(x, \rho, \Omega) e^{i \rho y+\Omega t}+\text { complex conjugate } \\
& \mathbf{v}(x, y, t)=\mathbf{V}(x, \rho, \Omega) e^{i \rho y+\Omega t}+\text { complex conjugate. }
\end{aligned}
$$

We have decomposed the $y$-dependence as $\exp (i \rho y)$, since the perturbations should be bounded as a function of $y$. Thus we consider all real values of $\rho$. Analogously, values of $\Omega$ with positive real part lead to unbounded behavior in $t$. If values of $\Omega$ with positive real part are found for a real value of $\rho$ and for bounded (to be made specific below) functions $N(x, \rho, \Omega)$ and $\mathbf{V}(x, \rho, \Omega)$, the corresponding line solution $\left(\eta^{*}, u^{*}, 0\right)$ is called spectrally unstable. If no such $\Omega$ exist, the line solution is spectrally stable. Note that the special case of $\rho=0$ corresponds to longitudinal or one-dimensional perturbations, whereas $\rho \neq 0$ corresponds to transverse perturbations.

Substituting the decomposition (1.8) into the linear equations (1.7) and denoting $\mathbf{V}=$ $(U, V)^{T}$, one obtains a system of ordinary differential equations of the form

$$
\begin{gathered}
\left(\begin{array}{ccc}
W L \partial_{x}+u_{x}^{*}+u^{*} \partial_{x} & M_{2} \partial_{x}+\eta_{x}^{*} & i \rho M_{2} \\
M_{1} \partial_{x} & W K \partial_{x}+u_{x}^{*}+u^{*} \partial_{x} & 0 \\
i \rho M_{1} & i \rho u^{*} & W K \partial_{x}
\end{array}\right)\left(\begin{array}{c}
N \\
U \\
V
\end{array}\right) \\
=\Omega\left(\begin{array}{ccc}
L & 0 & 0 \\
0 & K & 0 \\
0 & 0 & K
\end{array}\right)\left(\begin{array}{c}
N \\
U \\
V
\end{array}\right)
\end{gathered}
$$


where the operators $K, L, M_{1}$, and $M_{2}$ are defined as

$$
\begin{aligned}
K & =d\left(\partial_{x}^{2}-\rho^{2}\right)-1, & & L=b\left(\partial_{x}^{2}-\rho^{2}\right)-1, \\
M_{1} & =c\left(\partial_{x}^{2}-\rho^{2}\right)+1, & & M_{2}=a\left(\partial_{x}^{2}-\rho^{2}\right)+1+\eta .
\end{aligned}
$$

System (1.9) is a generalized (due to the presence of the diagonal matrix $\operatorname{diag}(L, K, K)$ on the right-hand side) spectral problem for the eigenfunction vector $(N, U, V)^{T}$. Note that for $b>0, d>0$ the generalized spectral problem is nonsingular, and it may be converted without obstruction to a standard spectral problem. All examples considered below will always fall into this category. Our goal is to determine the spectrum of $(1.9)$ for given $\left(\eta^{*}, u^{*}, 0\right)$. If we do so only for $\rho=0$, we obtain statements about the spectral (in)stability of our line solution with respect to longitudinal perturbations, whereas otherwise we are also considering transverse perturbations. Summarizing, we use the following definitions.

Definition 1.1. A line solution is longitudinally spectrally stable if for $\rho=0$ the spectrum of (1.9) does not intersect the right-half $\Omega$ plane.

Definition 1.2. The line solution is spectrally stable (longitudinally and transversally) if for all $\rho$ the spectrum of (1.9) does not intersect the right-half $\Omega$ plane.

A line solution is unstable with respect to the mode $(N, U, V)$ if the corresponding $\Omega$ has a positive real part. The instability is transversal if $\rho \neq 0$; otherwise it is longitudinal.

The study of (1.9) for a given $\eta^{*}$ and $u^{*}$ is the central topic of this paper. Clearly, the spectrum mentioned above depends on the boundary conditions imposed on the eigenfunction components $(N, U, V)$. These will be elaborated upon for each example given below.

2. Two numerical methods. Due to the complicated nature of the spectral stability problem (1.9), our investigations in this paper are strictly numerical. We begin by briefly discussing the two numerical methods employed.

2.1. Numerical method for studying cnoidal waves. If the coefficients in (1.9) are periodic, we use Hill's method [14]. It should be noted that (1.9), being a generalized spectral problem, is outside the realm of problems for which the convergence properties of Hill's method were analyzed in [12]. As remarked above, $\operatorname{since}$ the diagonal matrix $\operatorname{diag}(L, K, K)$ is nonsingular for $b>0, d>0$, the problem may be rewritten as a standard spectral problem, to which the techniques of [12] may be applied. It follows that our results are trustworthy, provided a sufficient number of Fourier modes are used in Hill's method.

Using Hill's method, we investigate perturbations of the periodic solutions that are bounded on the whole line. Our perturbations are not necessarily periodic and definitely do not need to share the same minimal period of the periodic solutions. In practice, a discrete set of Floquet exponents (see [14]) is used, resulting in perturbations that are periodic with period equal to an integer multiple of the period of the cnoidal wave solutions. In what follows, when a number of Fourier modes is listed as $N$, this implies that $2 N+1$ terms are present in the complex form of the Fourier series.

2.2. Numerical method for studying solitary waves. Hill's method is not intended for linear operators with coefficients that are localized on the whole line. As discussed in Appendix A, there are two ways to stretch the method to use it in this case: we may truncate (chop) the coefficient functions and periodically extend them, while considering increasingly 
larger periods. Alternatively, we approximate the localized function by an elliptic one which converges to it as the elliptic modulus approaches 1 . In essence, the two methods use the same principle of periodic approximations. The periodic approximation is smooth for the second approach which appears superior for this problem. For both approaches, the number of Fourier modes needs to be increased as the period increases, to ensure that Hill's method itself is providing an accurate approximation to the periodic problem. In all cases below where results using Hill's method are given, the second approach was used. The elliptic approximation function will be specified for each case given.

Alternatively, we use Hill's method using Hermite functions. This method is new, and it is outlined in detail in Appendix A.

3. Spectral stability of line solitary waves. In this section, we study the stability of line solitary waves.

3.1. Elevation solitary waves. Our first example examines a system which models surface waves where the system is globally well-posed and is known to be Hamiltonian $[3,4]$. The system has a one-parameter family of explicit solitary wave solutions resembling KdV solitons: the solution consists of a single localized disturbance with $\eta^{*}$ positive with a single maximum. Further, $\eta^{*}$ approaches 0 as $x \rightarrow \pm \infty$.

Although it is natural to believe from results from the theory of the KdV and KP (Kadomtsev-Petviashvili) equations that these solitary waves are stable, this is by no means a given: system (1.1) differs from the KdV and KP equations in many fundamental aspects. Perhaps the major difference is that system (1.1) is derived without the assumption that the waves travel in one direction and that they are nearly one-dimensional. It follows that energy can flow equally in all directions. Given the fundamental importance of solitary waves, these solutions seem a natural starting place for our stability investigations of (1.1).

Specifically, in (1.1) we use the parameter values

$$
a=-\frac{1}{9}, \quad b=\frac{1}{3}, \quad c=-\frac{1}{9}, \quad d=\frac{2}{9} .
$$

The line solution that we study is given by [8]:

$$
\begin{aligned}
& \eta^{*}=\eta_{0} \operatorname{sech}^{2}(\lambda x), \\
& u^{*}=\eta_{0} \sqrt{\frac{3}{\eta_{0}+3}} \operatorname{sech}^{2}(\lambda x),
\end{aligned}
$$

where

$$
\lambda=\frac{1}{2} \sqrt{\frac{2 \eta_{0}}{3(a-b)+2 b\left(\eta_{0}+3\right)}}
$$

and

$$
W=\frac{3+2 \eta_{0}}{\sqrt{3\left(3+\eta_{0}\right)}} .
$$

Here $\eta_{0}>0$ is a free parameter. 
Table 1

The apparent convergence of the eigenvalue with the largest real part of (1.9) with $\rho=0$ for increasing elliptic modulus $k$, approximating the elevation soliton (3.2) with $\eta_{0}=0.1$.

\begin{tabular}{c|c|c|c}
\hline Elliptic modulus $k$ & Period & Number of Fourier modes & Largest real part \\
\hline 0.8 & $<20$ & 20 & $2.97 \times 10^{-3}$ \\
0.9 & 20 & 20 & $2.14 \times 10^{-3}$ \\
0.99 & 28 & 20 & $6.87 \times 10^{-4}$ \\
0.999 & 37 & 30 & $2.18 \times 10^{-4}$ \\
0.999999 & 62 & 70 & $7.09 \times 10^{-6}$ \\
\hline
\end{tabular}

Using the Hermite method, no conclusive results are obtained: increasing the number of modes does not result in any apparent Cauchy convergence of the numerical results. It seems that for this case the difficulties presented by the essential spectrum are too much for the method to handle. We were able to do better using Hill's method, replacing the $\operatorname{sech}^{2}(\lambda x)$ by $\operatorname{cn}(\lambda x, k)$, where $\mathrm{cn}$ is the Jacobi elliptic cosine function [7] with elliptic modulus $k$. For fixed $\lambda, \operatorname{cn}(\lambda x, k) \rightarrow \operatorname{sech}(\lambda x)$ as $k \rightarrow 1$. The period of $\operatorname{cn}^{2}(\lambda x, k)$ is given by $2 K(k) / \lambda$, where $K(k)$ is the complete elliptic integral of the first kind [7],

$$
K(k)=\int_{0}^{\pi / 2} \frac{d z}{\sqrt{1-k^{2} \sin ^{2} z}} .
$$

For $\eta_{0}=0.1$ and $\rho=0$ (one-dimensional perturbations) the results are given in Table 1. It appears safe to conclude from these numerical results that the elevation solitary wave (3.2) is spectrally stable with respect to one-dimensional perturbations. Indeed, it seems that the solution (3.2) is spectrally stable with respect to one-dimensional perturbations for all amplitudes. Figure 1 compiles the results from several numerical experiments, displaying the positive real part of the spectrum of (1.9) with $\rho=0$ and $\eta_{0}$ varying from 0.1 to 1 in increments of 0.1 . The top panel shows the results obtained with $k=0.99$, whereas the bottom panel displays the same for $k=0.999$, with the number of Fourier modes increased accordingly. It is obvious from the figure that the spectrum corresponding to higher-amplitude solutions is harder to approximate numerically (requiring a higher elliptic modulus, i.e., a longer period), but increasing the elliptic modulus decreases all real parts consistently, allowing us to claim one-dimensional spectral stability for the elevation solitary wave (3.2). To show the further decrease in the value of the real parts, we computed the spectrum for $\eta_{0}=1$ with $k=0.999999$, resulting in a maximal real part of $1.31 \times 10^{-4}$. In contrast, the bottom panel of Figure 1 shows a corresponding value of $4.02 \times 10^{-3}$, showing a decrease by a factor of 30 using this more accurate (and time-consuming) computation.

Next, we examine the effect of $\rho \neq 0$; i.e., we investigate transverse perturbations. First, we fix the amplitude of the solution, letting $\eta_{0}=0.1$ and varying $\rho$ from 0 to 1 . Gradually increasing the elliptic modulus to obtain increasingly better approximations, we found no discernible real parts to the spectrum. Specifically, all real parts computed seemed to converge to zero as the elliptic modulus was increased. In other words, transverse perturbations do not change the stability results from the one-dimensional case. A similar statement is true for large-amplitude solutions: letting $\eta_{0}=0.9$, no change in the stability behavior from the onedimensional case is found as $\rho$ is increased away from zero. As expected, this calculation 

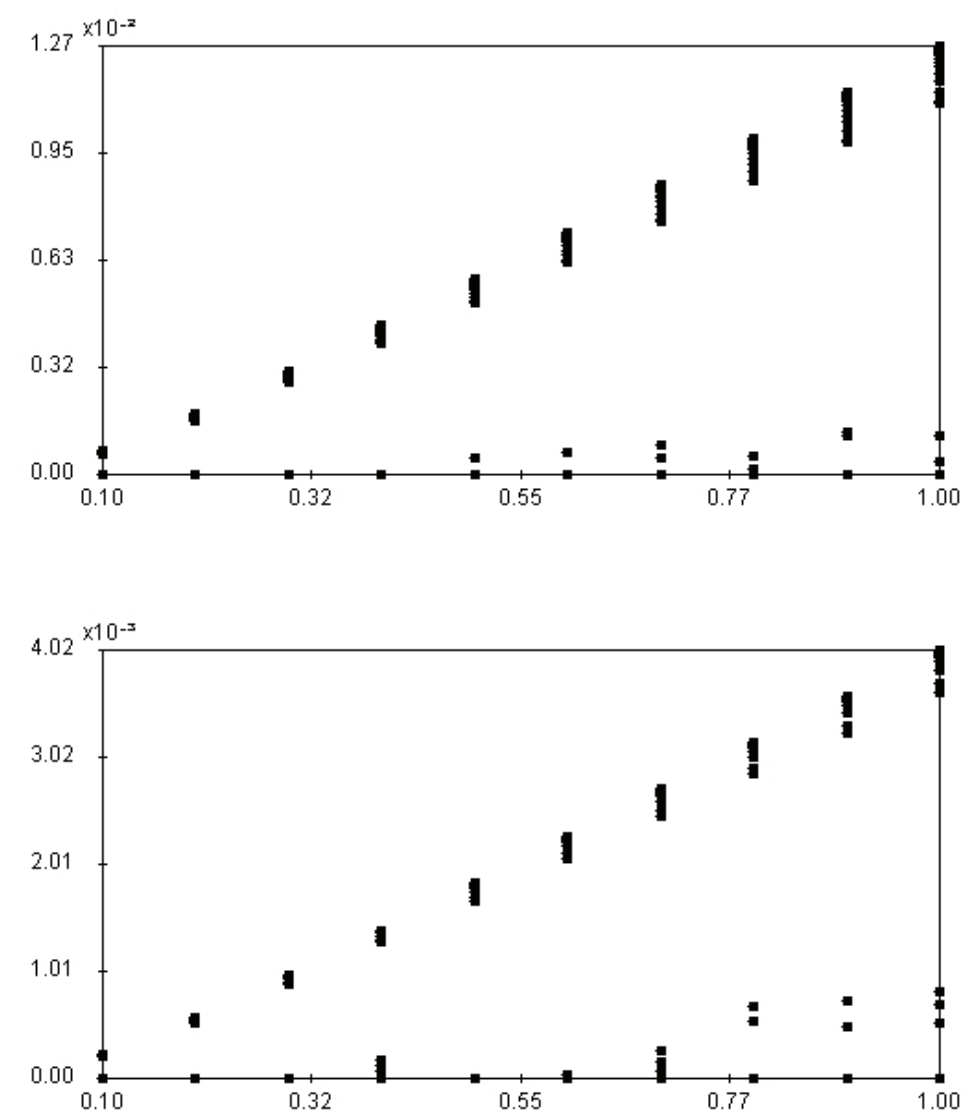

Figure 1. The positive real part of the spectrum of (1.9) with $\rho=0$ for amplitudes $\eta_{0} \in[0.1,1]$. For the top panel, $k=0.99$, whereas for the bottom panel, $k=0.999$. Roughly, the bottom panel equals the top panel, with all plotted $y$ values multiplied by three.

requires even higher elliptic moduli and a correspondingly larger number of Fourier modes to obtain results with real parts equally small as in the $\eta_{0}=0.1$ solution case.

3.2. Depression solitary waves. For a wide range of values of $a, b, c$, and $d$, we may consider the above solution with a negative value of $\eta_{0}$, with the range of $\eta_{0}$ limited by requiring reality of the solution. This gives rise to a depression solitary wave: a solitary wave approaching zero at infinity, with $\eta^{*}$ negative throughout, attaining a single minimum. In water wave tank experiments, any attempt to create a wave of depression results in an oscillatory wave train [16]. For certain parameter values $a, b, c$, and $d$, the Boussinesq system has explicit traveling depression solitary waves, even when no surface tension effects are included. In this section, we explore their stability or instability.

We use the parameter values

$$
a=-\frac{8}{9}, \quad c=-\frac{8}{9}, \quad b=\frac{10}{9}, \quad d=1 .
$$




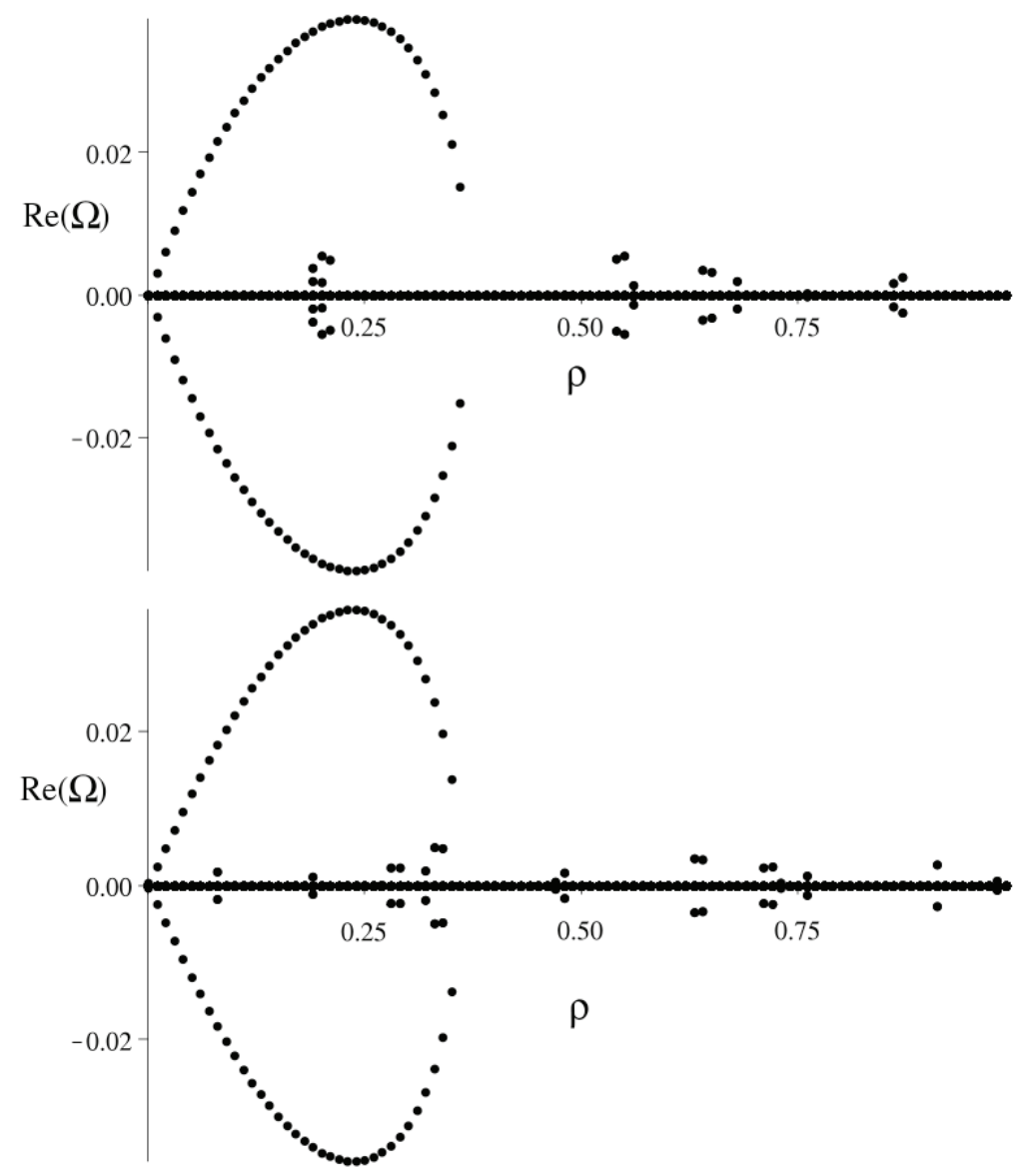

Figure 2. The real part of the spectrum of (1.9) as a function of $\rho$, for the depression soliton with $\eta_{0}=-1 / 2$. The computation of the top panel used 40 Hermite modes. For the bottom panel 59 modes were used.

Using the unscaled Hermite method (see Appendix A) with either 40 or 59 modes, we obtain Figure 2. These results indicate the spectral stability of the depression solitary wave with respect to one-dimensional $(\rho=0)$ perturbations, but they also demonstrate the presence of transverse instabilities. The most unstable transverse mode for our parameter values exists around $\rho=0.2$. It is unclear why the essential spectrum does not present the same difficulties for the Hermite method as it does for the elevation solitary wave case. Although not conclusive, the results of Figure 2 also indicate stability with respect to transverse perturbations of sufficiently short wave length (i.e., sufficiently large $\rho$ ). These results agree with our numerical experiments using Hill's method. The results for $\rho=0$ are shown in Table 2. Note that, due to the different parameters, the periods used for equal values of $k$ are smaller, reaching 22 for $k=0.999999$. Using Hill's method for $\rho=0.2$ with 70 modes for $k=0.99999999$, we determine the growth rate of the most unstable mode to be 0.0376 .

Similar results hold for $\eta_{0}=-1$, as seen in Table 3 . In this case, the most unstable mode occurs for $\rho$ near 0.3 . We find its growth rate to be 0.0718 . 
Table 2

The apparent spectral stability with respect to one-dimensional perturbations for the depression solitary wave with $\eta_{0}=-1 / 2$.

\begin{tabular}{c|c|c}
\hline Elliptic modulus $k$ & Number of Fourier modes & Largest real part \\
\hline 0.8 & 10 & 0.03 \\
0.9 & 15 & 0.0224 \\
0.99 & 20 & $7.58 \times 10^{-3}$ \\
0.999 & 30 & $2.42 \times 10^{-3}$ \\
0.999999 & 70 & $7.88 \times 10^{-5}$ \\
\hline
\end{tabular}

Table 3

The apparent spectral stability with respect to one-dimensional perturbations for the depression solitary wave with $\eta_{0}=-1$.

\begin{tabular}{c|c|c}
\hline Elliptic modulus $k$ & Number of Fourier modes & Largest real part \\
\hline 0.99 & 20 & $6.36 \times 10^{-2}$ \\
0.999 & 30 & $4.61 \times 10^{-3}$ \\
0.999999 & 70 & $1.5 \times 10^{-4}$ \\
\hline
\end{tabular}

3.3. Multipulse solutions. For some values of the parameters $a, b, c$, and $d$, the Boussinesq system (1.1) was shown in [8] to possess multipulse solutions: solutions with more than one maximum but still localized on the whole line. Actually, it was shown in [9] that such systems have multipulse solutions with any number of pulses. In this section we numerically investigate the spectral stability of some such solutions.

If we let $a=c=0$ and $b=d=1 / 6$, then one easily checks that

$$
\begin{aligned}
\eta^{*} & =\frac{15}{4}\left(-2+\cosh \left(\sqrt{\frac{18}{5}} x\right)\right) \operatorname{sech}^{4}\left(\frac{3 x}{\sqrt{10}}\right) \\
& =-\frac{45}{4} \operatorname{sech}^{4}\left(\frac{3 x}{\sqrt{10}}\right)+\frac{15}{2} \operatorname{sech}^{2}\left(\frac{3 x}{\sqrt{10}}\right), \\
u^{*} & =\frac{6}{5} \operatorname{sech}^{2}\left(\frac{3 x}{\sqrt{10}}\right)
\end{aligned}
$$

is a (two-pulse) solution. Using the unscaled Hermite method with 40 and 59 modes, respectively, we find the results displayed in Figure 3. These numerical results clearly indicate both the one-dimensional and transverse instability of the multipulse solution (3.7). For the case of one-dimensional perturbations, we also employed Hill's method, by using $\mathrm{cn}(\cdot, k)$ to approximate $\operatorname{sech}(\cdot)$ in (3.7). The results of these computations are given in Table 4. They show perfect agreement with those of Figure 3.

4. Spectral stability of line cnoidal waves. In this section, we study the stability of two cnoidal wave solutions of the Boussinesq system with $a=c=0, b=d=1 / 6$. Both solutions are examples of the cnoidal wave solutions constructed in [10]. As stated in the introduction, an alternate method for constructing the cnoidal wave solutions is discussed in Appendix B. Since these solutions are periodic, we rely solely on Hill's method for numerical results. We restrict ourselves to a small number of numerical experiments, since these already demonstrate the spectral instability of the cnoidal wave solutions. 


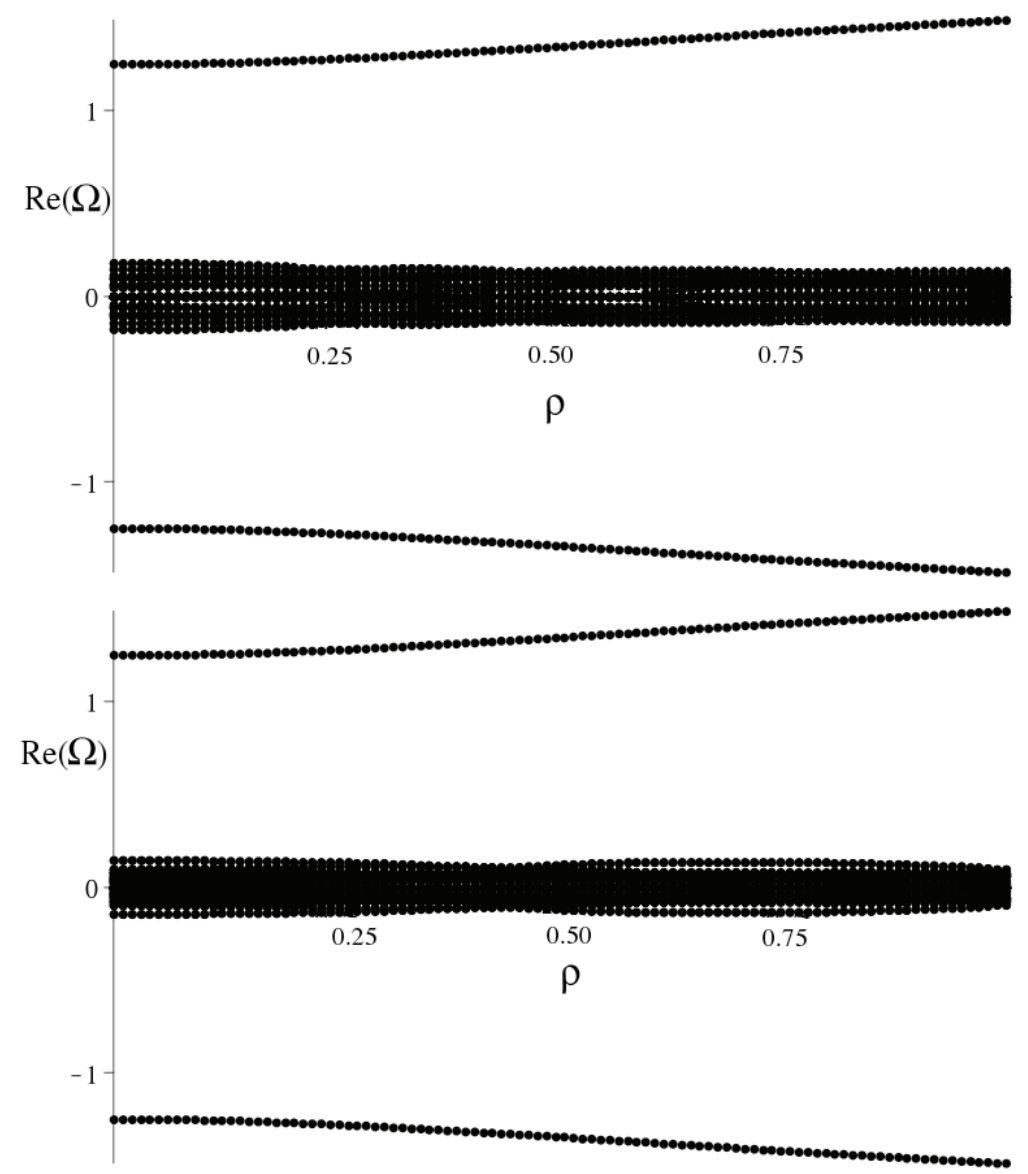

Figure 3. Real part of the spectrum of (1.9) as a function of the transverse perturbation wave number $\rho$ for the multipulse solution (3.7), using 40 Hermite modes (top) and 59 Hermite modes (bottom).

Table 4

Approximating the largest real part of the spectrum of (1.9) for $\rho=0$ for the two-pulse solution (3.7) using Hill's method.

\begin{tabular}{c|c|c|c}
\hline Elliptic modulus $k$ & Period & Number of Fourier modes & Largest real part \\
\hline 0.9 & 5.4 & 20 & 1.28 \\
0.99 & 7.8 & 30 & 1.25 \\
0.999 & 10.2 & 30 & 1.25 \\
0.9999 & 12.6 & 50 & 1.2495363 \\
0.999999 & 17.4 & 70 & 1.2494668 \\
\hline
\end{tabular}

Case 1. Consider the solution

$$
\begin{aligned}
& u^{*}=-0.7484+3.5656 \mathrm{cn}^{2}(1.9642 x, 0.94868), \\
& \eta^{*}=0.30806 u^{*}-\frac{1}{2} u^{* 2}-\frac{0.30806}{6} 3.858 u_{x x}^{*} .
\end{aligned}
$$

Using Hill's method with 20 Fourier modes, we find the spectrum shown in Figure 4. It clearly demonstrates the spectral instability of the solution (4.1). A large variety of instabilities 

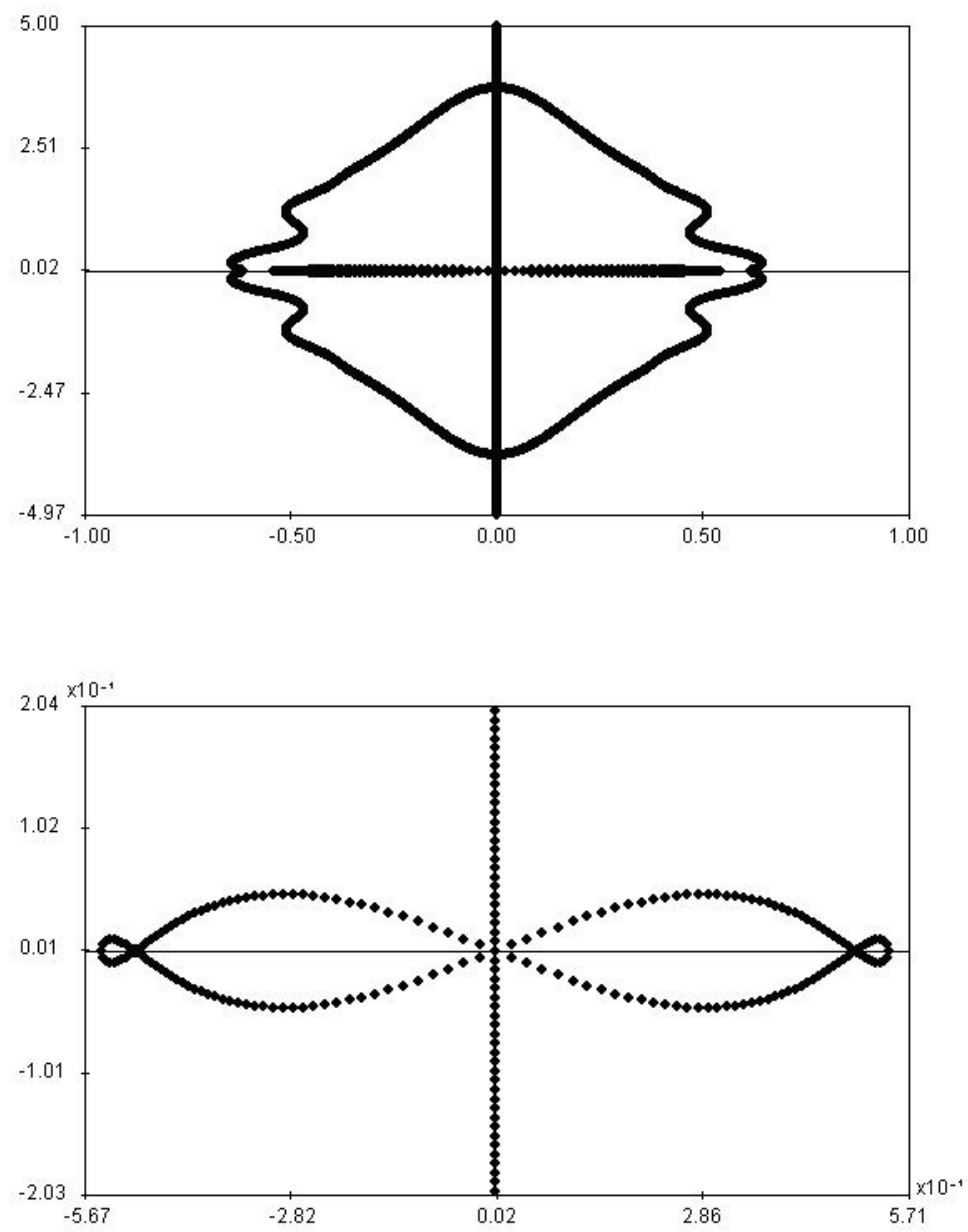

Figure 4. The spectrum of (1.9) for the cnoidal-wave solution (4.1), using 20 Fourier modes (top), and a blow-up of the region around the origin (bottom).

can be seen in Figure 4. The bottom panel clearly shows the presence of a modulational instability (growth of modes with periods almost equal to that of the cnoidal wave solution): the eigenvalue at the origin corresponds to eigenfunctions of the same period. Nearby points have eigenfunctions with slightly different periods, as well as nonzero frequency instabilities (i.e., $\Omega$ has nonzero imaginary part).

Case 2. Next we consider

$$
\begin{aligned}
u^{*} & =0.4303+7.2355 \mathrm{cn}^{2}(0.96793 x, 0.94868), \\
\eta^{*} & =0.30806 u^{*}-\frac{1}{2} u^{* 2}-\frac{0.30806}{6} 3.858 u_{x x}^{*} .
\end{aligned}
$$

The spectrum for this case is shown in Figure 5. Both cases are already unstable with respect to one-dimensional perturbations. We did not investigate the effect of transverse perturbations. 


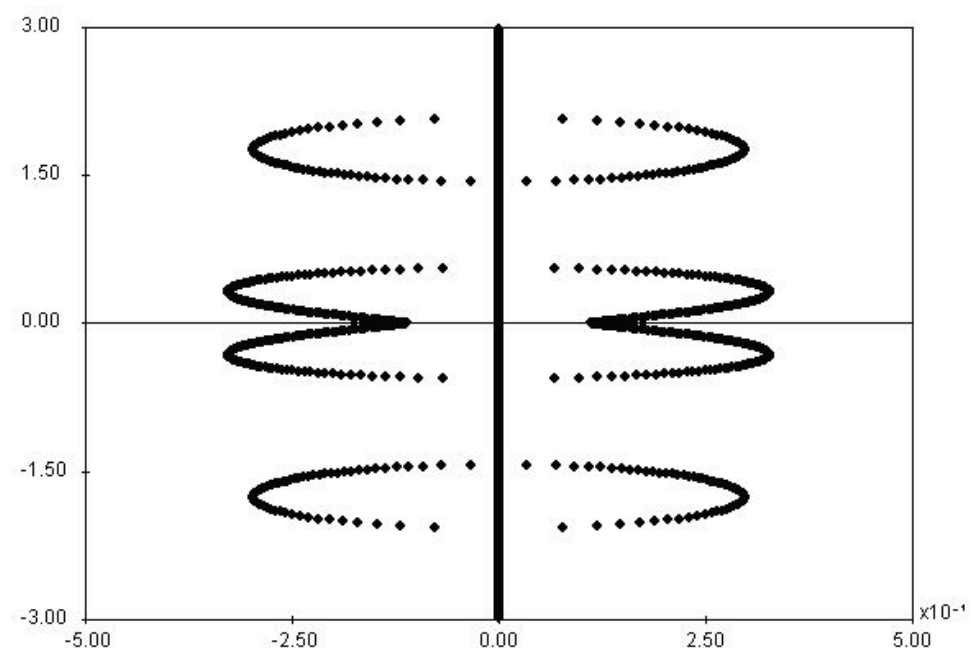

Figure 5. The spectrum of (1.9) for the cnoidal-wave solution (4.2), using 20 Fourier modes.

5. Conclusions. We have investigated the spectral stability of different types of line (one-dimensional) solutions of the Boussinesq system (1.1). Our results are summarized as follows:

- Elevation solitary waves. We have demonstrated numerically that elevation solitary waves are stable with respect to both one-dimensional perturbations and transverse perturbations.

- Depression solitary waves. Numerical experiments indicate that depression solitary waves are stable with respect to one-dimensional perturbations, but unstable with respect to transverse perturbations.

- Multipulse solitary waves. In contrast to one-pulse elevation solitary waves, we found that multipulse solitary waves are unstable, even with respect to one-dimensional perturbations. Adding transverse instabilities increases the growth rates of the unstable modes.

- Cnoidal waves. Cnoidal waves are found to be unstable with respect to a plethora of one-dimensional perturbations. No numerical exploration of transverse perturbations was undertaken.

What is the impact of these results for the validation of the Boussinesq system (1.1)? Our results for the solitary wave solutions agree, at least qualitatively, with those of other modeling equations such as the KdV equation. Therefore, we focus on the stability behavior of periodic solutions. Recent work (see $[6,13]$ ) has established the spectral stability of the cnoidal wave solutions of the $\mathrm{KdV}$ equation with respect to bounded perturbations, and their orbital stability with respect to perturbations that are periodic with period equal to an integer multiple of the cnoidal wave period. Thus our results disagree with those in the context of the $\mathrm{KdV}$ equation. For the full Euler water wave problem, different numerical results [15, 18, 19] demonstrate the instability of periodic waves in shallow water. We conclude that at least this 
aspect of the water wave problem is better described by the Boussinesq system, as opposed to, for instance, the $\mathrm{KdV}$ equation.

Appendix A. The numerical computation of spectra of linear operators with localized coefficients. In [14] a method was discussed which efficiently and accurately computes spectra of linear operators with periodic coefficients. The method may be pushed to problems with coefficients defined on all of $\mathbb{R}$ using one of two scenarios:

- The operator may be considered on a finite interval and periodically extended. The convergence of this truncation process can be judged by consideration of increasingly longer intervals.

- We may replace the coefficient functions of the operator by periodic ones which limit to the whole-line ones as their period increases. For instance, hyperbolic functions may be replaced by elliptic ones.

Both scenarios prove to be useful. The second one turns out to be especially effective for the computation of our spectra, as is discussed in the examples in the main text. Nevertheless, it would be convenient to have at our disposal a method which investigates whole-line problems directly, without the need to investigate the limit from a periodic case. To this end, we present here a Galerkin method using Hermite functions, the set of which is orthonormal and complete in $L^{2}(\mathbb{R})$.

Recall that the $n$th Hermite function is defined as [1]

$$
\mathrm{e}_{n}=\frac{1}{\sqrt{2^{n} \sqrt{\pi} n !}} e^{-x^{2} / 2} H_{n}
$$

where $H_{n}$ is the $n$th Hermite polynomial. Considering a generalized $N \times N$ spectral problem

$$
\mathbf{S} \phi=\Omega \mathbf{M} \phi,
$$

where $\phi$ is a vector of complex-valued functions, $\mathbf{S}$ is a matrix of differential operators $S_{j}^{i}$, as is $\mathbf{M}$ (components $M_{j}^{i}$ ). In addition, we require $M$ to be invertible as a linear operator. The scalar $\Omega$ is the spectral parameter. Using Einstein's summation convention, we rewrite this problem as

$$
S_{j}^{i} \phi^{j}=\Omega M_{j}^{i} \phi^{j} .
$$

Denote the expansion in terms of the basis functions $\mathrm{e}_{n}$ for each $\phi^{j}$ as

$$
\phi^{j}(x)=\sum_{m=0}^{\infty} \hat{\phi}^{j, m} \mathrm{e}_{m} .
$$

Taking the $\mathrm{L}_{2}$ inner product of (A.3) with $\mathrm{e}_{n}$ and expanding each $\phi^{j}$, we have

$$
\sum_{m=0}^{\infty} \hat{\phi}^{j, m}\left\langle S_{j}^{i} \mathrm{e}_{m}, \mathrm{e}_{n}\right\rangle=\Omega \sum_{m=0}^{\infty} \hat{\phi}^{j, m}\left\langle M_{j}^{i} \mathrm{e}_{m}, \mathrm{e}_{n}\right\rangle .
$$

Define

$$
\widehat{M}_{j, m}^{i, n} \equiv\left\langle M_{j}^{i} \mathrm{e}_{m}, \mathrm{e}_{n}\right\rangle, \quad \widehat{S}_{j, m}^{i, n} \equiv\left\langle S_{j}^{i} \mathrm{e}_{m}, \mathrm{e}_{n}\right\rangle
$$


This allows us to write (A.5) as

$$
\sum_{m=0}^{\infty} \widehat{S}_{j, m}^{i, n} \hat{\phi}^{j, m}=\Omega \sum_{m=0}^{\infty} \widehat{M}_{j, m}^{i, n} \hat{\phi}^{j, m} .
$$

This equation corresponds to a generalized eigenvalue problem involving two infinite-dimensional matrices. Our numerical method for computing approximations for $\Omega$ is obtained by truncating the infinite sums in (A.7) at a sufficiently large value, say $N$, which results in

$$
\sum_{m=0}^{N} \widehat{S}_{j, m}^{i, n} \hat{\phi}^{j, m}=\Omega \sum_{m=0}^{N} \widehat{M}_{j, m}^{i, n} \hat{\phi}^{j, m} .
$$

In effect, we are introducing a projection along the first $N$ Hermite functions, demonstrating that this is indeed a Galerkin method. Last, a standard eigenvalue solver produces the desired approximation of the spectrum.

A major difficulty in implementing this method is the determination of the coefficients (A.6), unlike in the periodic case where Fourier modes are used. In general, when using Hermite functions, it is not possible to find a closed-form expression for these terms due to the presence of nonconstant function coefficients in the linear operators. We need to find accurate approximations to expressions like

$$
\left\langle f(x) \mathrm{e}_{m}, \mathrm{e}_{n}\right\rangle .
$$

We could attempt to numerically integrate the above expression. However, if we use a truncation value of $N$ in (A.8), the function $f(x)$ is integrated against modes like $e_{2 N}(x)$. For large $N$, the rapid oscillations of the Hermite functions lead to catastrophic cancellation in our numerical integration. Thus, we first approximate $f(x)$ so that our choice of $N$ is not limited by this effect.

We proceed as follows. Assuming

$$
f(x)=\sum_{l=0}^{\infty} \hat{f}_{l} \mathrm{e}_{l},
$$

we write

$$
\left\langle f(x) \mathrm{e}_{m}, \mathrm{e}_{n}\right\rangle=\sum_{l=0}^{\infty} \hat{f}_{l}\left\langle\mathrm{e}_{l} \mathrm{e}_{m}, \mathrm{e}_{n}\right\rangle .
$$

The contributions $\left\langle\mathrm{e}_{l} \mathrm{e}_{m}, \mathrm{e}_{n}\right\rangle$ can, in principle, be computed analytically. Further, if

$$
f(x)=O\left(e^{-p|x|}\right)
$$

as $|x| \rightarrow \infty$ for some positive constant $p$, and if $f(x)$ is analytic on the strip $|\operatorname{Im}(x)| \leq \omega$, then

$$
\hat{f}_{l}=O\left(e^{-\omega \sqrt{2 l+1}}\right)
$$


as $l \rightarrow \infty$. Here $\omega=\min (p, \tau)$, where $\tau$ is the magnitude of the imaginary part of the nearest singularity of $f(x)$ to the real axis [21]. For our purposes, $f(x)$ will always satisfy this constraint with $\omega=\pi / 2 \lambda$. The terms $\left\langle\mathrm{e}_{l} \mathrm{e}_{m}, \mathrm{e}_{n}\right\rangle$ decay even faster than $\hat{f}_{l}$, and therefore the finite-sum truncation of (A.11) incurs only an exponentially small error. Thus we safely use

$$
\tilde{f}=\sum_{l=0}^{M} \hat{f}_{l} \mathrm{e}_{l} .
$$

In most problems that we study, we have (perhaps after some rewriting) $f(x)$ to be $\operatorname{sech}^{2}(\alpha x)$, where $\alpha$ is real. In any case where a derivative of $f(x)$ is needed, we first use integration by parts on

$$
\left\langle f^{\prime}(x) \mathrm{e}_{m}, \mathrm{e}_{n}\right\rangle
$$

and use the identity

$$
\frac{d \mathrm{e}_{n}}{d x}=\sqrt{\frac{n}{2}} \mathrm{e}_{n-1}-\sqrt{\frac{n+1}{2}} \mathrm{e}_{n+1}
$$

to get a new analytic expression involving only $f(x)$. While this makes implementing the algorithm slightly more complicated, it minimizes the amount of numerical integration needed. Even so, Maple 12 allows for a reliable and accurate approximation of $\left\langle\operatorname{sech}^{2}(\alpha \xi), \mathrm{e}_{l}\right\rangle$ only for $l \leq 59$. Thus we use $M \leq 59$ throughout, for all problems where we use the Hermite method in this paper.

Remarks.

- The spectrum of differential operators with localized coefficients which decay sufficiently fast at infinity consists of the essential spectrum (easily calculated, as its determination requires one to solve a problem with constant coefficients [20]) and the discrete spectrum consisting of eigenvalues. Given the ease of determining the essential spectrum, we are mainly interested in a numerical method which accurately determines the discrete spectrum. Our choice of the Hermite functions as basis functions immediately indicates that we should expect good approximations to the eigenvalues and their associated square-integrable eigenfunctions. We investigate this in the examples below.

- The convergence properties of Hill's method using Fourier series were examined numerically in [14]. More recently, the analysis of the effect of the truncation to a finite number of modes was undertaken in [12], where different convergence theorems were proven. No such analysis has been undertaken for the Hermite method.

- In order to improve the accuracy of the approximation (A.14), we may use a scaled form of the Hermite functions to take into account the scaling $\alpha$ in $\operatorname{sech}^{2}(\alpha x)$. Thus, instead of $\mathrm{e}_{n}$, we use $\sqrt{\gamma} \mathrm{e}_{n}(\gamma x)$, where $\gamma=\sqrt{2} \alpha$. The effect of using this scaling can be seen in Figure 6, which shows the absolute value of the difference between $\operatorname{sech}^{2}(x / 2)$ and (A.14) using both unscaled and scaled Hermite functions. It is clear that in this case using the scaling improves the accuracy of the approximation by about two orders of magnitude. 


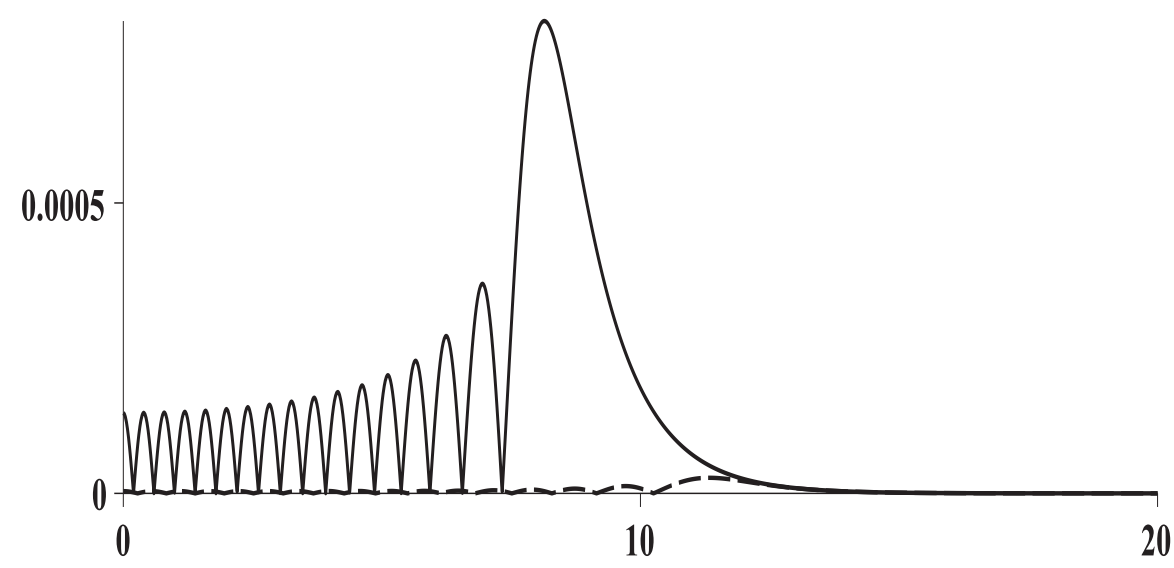

Figure 6. Approximation error for $\operatorname{sech}^{2}(x / 2)$ using unscaled Hermite functions (solid line) and scaled Hermite functions (dashed line), both with $N=31$.

However, while it appears that the above scaling often (but not always) improves the accuracy of (A.14), this does not necessarily imply that the scaled Hermite functions yield a better approximation to the spectrum of a given operator. When approximating a discrete eigenvalue, ideally we would scale the Hermite functions with respect to the width of the unknown corresponding eigenfunction.

As for the essential spectrum, as remarked above, the Hermite functions, regardless of scaling, are a poor means of approximating "eigenfunctions" associated with an element of the essential spectrum, as such functions cannot be localized. Thus we can only hope to obtain good approximations to the discrete spectrum and the associated localized eigenfunctions.

- The choice of Hermite functions as an orthonormal basis is not the only avenue for building a Galerkin method on the whole line. For instance, alternatively we might have used normalized Hermite functions with Gaussian measure in the norm. The problem with this approach is that it allows for eigenfunctions that do not decay at infinity, since the Gaussian measure allows such nonlocalized bounded functions to have finite norm. This is unfortunate, since we are able to derive closed-form expressions for (A.9) using the Gaussian measure.

To establish confidence in the method, we present some results on problems for which the spectrum is analytically known.

Example: The KdV equation. We write the KdV equation as

$$
u_{t}+3 u u_{x}+u_{x x x}=0,
$$

which has the soliton solution $u(x, t)=U(x-t)=\operatorname{sech}^{2}(x-t) / 2$. Linearizing around this solution results in the associated linear problem

$$
L \phi=\phi_{x x x}+3(U(x) \phi)_{x}-\phi_{x}=\lambda \phi .
$$

It is straightforward to show that the essential spectrum $\sigma_{\text {ess }}(L)=i \mathbb{R}$. It is shown in [17] that $L$ has no eigenvalues other than at zero due to symmetry. Thus $\sigma(L)=i \mathbb{R}$. We might wonder 
Table 5

Maximum of the real part of approximation to the spectrum, using a Maple implementation of the Hermite algorithm for the linear problem (A.18). A stated value of 0 . indicates that the result was indistinguishable from the software-induced precision of $10^{-15}$.

\begin{tabular}{c|c|c}
\hline Number of Hermite modes & $\max \operatorname{Re}(\lambda)$ (unscaled) & $\max \operatorname{Re}(\lambda)$ (scaled) \\
\hline 30 & $.198167349 \times 10^{-2}$ & 0. \\
40 & $.613955717 \times 10^{-3}$ & 0. \\
50 & $.222354504 \times 10^{-3}$ & 0. \\
59 & $.401588484 \times 10^{-15}$ & 0. \\
\hline
\end{tabular}

Table 6

Approximations to the discrete spectrum of (A.20), using a Maple implementation of the Hermite algorithm.

\begin{tabular}{c|c|c}
\hline Number of Hermite modes & $-\lambda_{N, 1}$ & $-\frac{3}{2}-\lambda_{N, 2}$ \\
\hline 10 & $.726725478 \times 10^{-4}$ & $.1334677136 \times 10^{-1}$ \\
20 & $.608876104 \times 10^{-6}$ & $.1108871556 \times 10^{-2}$ \\
30 & $.106195049 \times 10^{-7}$ & $.1575614131 \times 10^{-3}$ \\
40 & $.456898436 \times 10^{-9}$ & $.3004943429 \times 10^{-4}$ \\
50 & $.946901283 \times 10^{-10}$ & $.6932114778 \times 10^{-5}$ \\
59 & $.763100964 \times 10^{-10}$ & $.2372154196 \times 10^{-5}$ \\
\hline
\end{tabular}

what results our method will produce, given that we are applying it to something for which it is ill suited, namely approximating the essential spectrum. Table 5 shows the maximum value of the real part of the approximated spectrum using unscaled and scaled Hermite functions.

The eigenvector associated with the zero eigenvalue is the derivative of $U(x)$ and thus has the same scaling as $u$. Presumably, this explains the dramatic difference between approximating the spectrum with scaled and unscaled Hermite functions.

Example: The Allen-Cahn equation. An example involving a discrete spectrum originates from the Allen-Cahn equation

$$
u_{t}-u_{x x}-u+u^{3}=0
$$

with solution $u(x, t)=U(x)=\tanh (x / \sqrt{2})$. This gives rise to the linearization

$$
L \phi=\phi_{x x}-2 \phi+3 \operatorname{sech}^{2}\left(\frac{x}{\sqrt{2}}\right) \phi=\lambda \phi .
$$

Clearly, the operator $L$ is self-adjoint. It is easy to show that the essential spectrum $\sigma_{\text {ess }}(L)=$ $(-\infty,-2]$. We know that zero is an eigenvalue due to the translational symmetry of the AllenCahn equation. Further, using the transformation $\xi=\tanh (x / \sqrt{2})$, one transforms $L$ into an associated Legendre equation [2], which immediately gives that $-3 / 2$ is the only other eigenvalue of $L$. Thus $\sigma(L)=(-\infty,-2] \cup\{-3 / 2\} \cup\{0\}$.

Focusing on the eigenvalues $-3 / 2$ and 0 , if we take the smallest and next smallest eigenvalues in magnitude of our approximation, which for each $N$ we denote as $\lambda_{N, 1}$ and $\lambda_{N, 2}$, respectively, we obtain Table 6 .

Observe that the method produces a rapidly converging approximation to the true eigenvalues 0 and $-3 / 2$. Likewise, Figure 7 shows the output of our method using $N=59$, where 


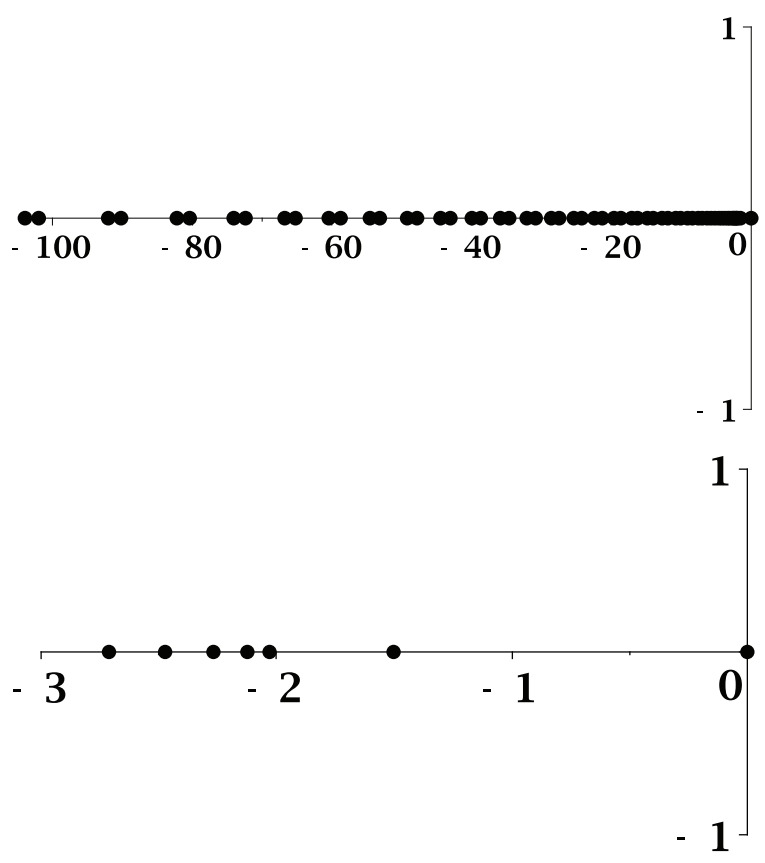

Figure 7. The approximated spectrum in the complex $\lambda$ plane for the Allen-Cahn problem, using $N=59$ Hermite modes. The bottom panel is an enlargement of the region close to the origin of the $\lambda$ plane of the top panel.

the bottom figure is a zoom-in near the origin of the top figure. It is clear that our method produces approximations at $-3 / 2,0$, and values in the interval $(-\infty,-2]$. This illustrates that using the Hermite algorithm is impractical for computing the essential spectrum, but it appears well suited for computing the discrete spectrum of an operator. We do not consider a scaling of the Hermite functions for this problem: the scaling we use for the Allen-Cahn problem would introduce a scaling on the Hermite functions equal to unity.

Appendix B. A new representation for the cnoidal wave solutions. In [10], an explicit representation was obtained for a large class of stationary solutions of (1.1). This representation, which is used in the main body of the present paper, is in terms of Jacobi elliptic functions [1]. Following the method of Conte and Musette [11], we obtain a different representation in terms of Weierstrass elliptic functions [1]. Another advantage of this approach is that we are able to show that the solutions found comprise all stationary elliptic solutions of the Boussinesq system (1.1).

The system of equations we are considering is

$$
\begin{aligned}
& -W \eta+u(1+\eta)+\frac{W}{6} \Lambda^{2} \eta^{\prime \prime}=0, \\
& -W u+\eta+\frac{1}{2} u^{2}+\frac{W}{6} \Lambda^{2} u^{\prime \prime}=0 .
\end{aligned}
$$

Here the prime denotes differentiation with respect to $\xi=\Lambda(x-W t)$, and $\Lambda$ represents a scaling. It could be omitted and reintroduced after the fact, using the scaling symmetry of the 
equations. The system of equations (B.1)-(B.2) is obtained by using the translating stationary profile ansatz, followed by integrating once with respect to $\xi$. The resulting integration constants are equated to zero. This is further discussed in [10].

Solving (B.2) for $\eta$ and substituting into (B.1) result in a single equation to be solved for $u$. It is on this equation that we use the method of Conte and Musette [11] to find all elliptic solutions of the scalar equation. This results in a rather messy description of the solutions, but with a simple functional form. Having accomplished this, we start directly from the found functional form with parameters. This ansatz is substituted into the scalar equation, and the parameters are determined. Our ansatz, determined using Conte and Musette's method, is

$$
u=\alpha+\beta \wp\left(\delta \xi+i \gamma, g_{2}, g_{3}\right)
$$

where $\wp\left(\delta \xi, g_{2}, g_{3}\right)$ denotes the Weierstrass elliptic function with invariants $g_{2}$ and $g_{3}$. Thus far $\alpha, \beta, \delta$, and $\gamma$ are undetermined parameters. Substitution into the scalar equation for $u$ allows us to find that

$$
\begin{aligned}
\alpha & =W \\
\beta & =-\frac{10}{3} \Lambda^{2} \delta^{2} W \\
g_{2} & =\frac{9\left(2+W^{2}\right)}{11 W^{2} \Lambda^{4} \delta^{4}}, \\
g_{3} & =-\frac{27}{20 \Lambda^{2} \delta^{6} W^{2}} .
\end{aligned}
$$

Note that the parameters $\delta$ and $\gamma$ are free. This was to be expected: $\delta$ reflects the scaling symmetry of the Boussinesq system, as does $\Lambda$. This explains why both can be grouped into a single parameter, $\Lambda \delta$. Last, $\gamma$ reflects the translational symmetry: the Boussinesq system is autonomous in $x$. However, if we wish to consider real and nonsingular solutions, $\gamma$ is not entirely arbitrary: its real part should be chosen to be half of the period of the Weierstrass function in the imaginary direction; see [1].

The class of solutions described in terms of the Weierstrass elliptic functions is identical to that given in [10]. Two advantages are obtained from our approach:

- Since we have followed the method of Conte and Musette [11], we learn that the solutions found in [10] are all the elliptic solutions of (B.1). This information could not be obtained by using an ansatz as in [10].

- To obtain an explicit numerical description of an elliptic solution using the description of [10] requires the solution of a cubic equation. Our description results in a far simpler parametrization of the solution branch: all parameters are determined explicitly in terms of the free parameters.

\section{REFERENCES}

[1] M. Abramowitz and I. A. Stegun, eds., Handbook of Mathematical Functions, With Formulas, Graphs and Mathematical Tables, National Bureau of Standards, Washington, DC, 1964.

[2] G. Arfken and H. J. Weber, Mathematical Methods for Physicists, 4th ed., Academic Press, New York, 1996. 
[3] J. L. Bona, M. Chen, And J.-C. SAut, Boussinesq equations and other systems for small-amplitude long waves in nonlinear dispersive media. I. Derivation and linear theory, J. Nonlinear Sci., 12 (2002), pp. $283-318$

[4] J. L. Bona, M. Chen, And J.-C. SAUt, Boussinesq equations and other systems for small-amplitude long waves in nonlinear dispersive media. II. The nonlinear theory, Nonlinearity, 17 (2004), pp. 925-952.

[5] J. Bona, T. Colin, And D. Lannes, Long wave approximations for water waves, Arch. Ration. Mech. Anal., 178 (2005), pp. 373-410.

[6] N. Bottman And B. Deconinck, Kdv cnoidal waves are linearly stable, Discrete Contin. Dynam. Systems, 25 (2009), 1163-1180.

[7] P. F. Byrd and M. D. Friedman, Handbook of Elliptic Integrals for Engineers and Scientists, SpringerVerlag, New York, 1971.

[8] M. Chen, Exact traveling-wave solutions to bi-directional wave equations, Internat. J. Theoret. Phys., 37 (1998), pp. 1547-1567.

[9] M. Chen, Solitary-wave and multi-pulsed traveling-wave solutions of Boussinesq systems, Appl. Anal., 75 (2000), pp. 213-240.

[10] H. Chen, M. Chen, And N. V. NGuyen, Cnoidal wave solutions to Boussinesq systems, Nonlinearity, 20 (2007), pp. 1443-1461.

[11] R. Conte and M. Musette, The Painlevé Handbook, Springer, Dordrecht, The Netherlands, 2008.

[12] C. Curtis And B. Deconinck, On the convergence of Hill's method, Math. Comp., 79 (2010), pp. 169-187.

[13] B. Deconinck And T. Kapitula, On the orbital (in)stability of spatially periodic stationary solutions of generalized Korteweg-de Vries equations, SIAM J. Appl. Anal., submitted.

[14] B. Deconinck And J. N. Kutz, Computing spectra of linear operators using Hill's method, J. Comput. Phys., 219 (2006), pp. 296-321.

[15] M. Francius And C. Kharif, Three-dimensional instabilities of periodic gravity waves in shallow water, J. Fluid Mech., 561 (2006), pp. 417-437.

[16] J. L. Hammack And H. Segur, The Korteweg-de Vries equation and water waves. III. Oscillatory waves, J. Fluid Mech., 84 (1978), pp. 337-358.

[17] A. Jeffery And T. Kakutani, Stability of Burgers shock wave and the Korteweg-de Vries soliton, Indiana Univ. Math. J., 20 (1970), pp. 463-468.

[18] J. W. MCLEan, Instabilities of finite-amplitude gravity waves on water of finite depth, J. Fluid Mech., 114 (1982), pp. 331-341.

[19] K. Oliveras, Stability of Periodic Surface Gravity Waves, Ph.D. thesis, Department of Applied Mathematics, University of Washington, Seattle, WA, 2009.

[20] B. SAndstede, Stability of travelling waves, in Handbook of Dynamical Systems, Vol. 2, North-Holland, Amsterdam, 2002, pp. 983-1055.

[21] G. Szegö, Orthogonal Polynomials, 4th ed., American Mathematical Society, Providence, RI, 1975. 